\title{
16. PRELIMINARY DATING BY FOSSIL CALCAREOUS NANNOPLANKTON, DEEP SEA DRILLING PROJECT: LEG 10
}

William W. Hay, Rosenstiel School of Marine and Atmospheric Science, University of Miami, Miami, Florida; Department of Geology, University of Illinois, Urbana, Illinois

\section{INTRODUCTION}

The zonation and ages used in this report correspond to those of Martini and Worsley (1970) for the Neogene, Martini (1970) for the Paleogene, and Cepek and Hay (1969) for the Late Cretaceous. The Gephyrocapsa oceanica Zone, defined by the first occurrence of the name species at the base and by the first occurrence of Emiliania huxleyi at the top, is used here instead of the Pseudoemiliania lacunosa Zone.

\section{HOLE 85/85A}

Samples: 85-1-1 (6-7 cm); 85-1-2 (6-7 cm); 85-1-3 $(3-4 \mathrm{~cm})$ : These samples contain generally poor assemblages; mostly reworked Late Cretaceous species, including Micula staurophora, Watznaueria barnesae, Arkhangelskiella octoradiata, Cribrosphaera ehrenbergi, Cretadiscus colatus, and Eiffellithus turriseiffeli. Some specimens of Gephyrocapsa aperta are present.

Age: Probably Gephyrocapsa oceanica Zone; Late Pleistocene.

Sample 85-1-4 $(6-7 \mathrm{~cm})$ :

Very poor assemblage with only Micula staurophora and a few corroded coccoliths.

Sample 85-1-5 $(10-11 \mathrm{~cm})$ :

Poor assemblage with some reworked Late Cretaceous species, as in Sample 85-1-3 $(3-4 \mathrm{~cm})$, but also including Gephyrocapsa caribbeanica? and G. aperta.

Age: Probably Gephyrocapsa oceanica Zone; Late Pleistocene.

Sample 85-1-6 $(6-7 \mathrm{~cm})$ :

Very poor assemblage with only a few specimens of Gephyrocapsa aperta and G. caribbeanica.

Age: Probably Gephyrocapsa oceanica Zone; Late Pleistocene.

Samples: 85-2-1 $(6-7 \mathrm{~cm}) ; 85-2-2(7-8 \mathrm{~cm})$ :

No calcareous nannofossils.

Sample 85-2-3 (14-15 cm):

Very poor assemblage with reworked species from Late Cretaceous, as in Sample 85-1-3 $(3-4 \mathrm{~cm})$, and ? Gephyrocapsa caribbeanica.

Age: Pleistocene.

Sample 85-3-1 $(10-11 \mathrm{~cm})$ :

Very poor assemblage with rare coccoliths, including Prediscosphaera cretacea from the Late Cretaceous, and Gephyrocapsa oceanica.

Age: Gephyrocapsa oceanica Zone; Late Pleistocene.

Samples: 85-3-2 (10-11 cm); 85-3-3 (7-8 cm); 85-3-4 (5-4 $\mathrm{cm})$ :

No calcareous nannofossils.
Samples: 85-3-6 (6-7 cm); 85-4-2 (10-11 cm); 85-5-1 (63-64 $\mathrm{cm})$ :

Very poor assemblages with Gephyrocapsa aperta and G. caribbeanica.

Age: Probably Gephyrocapsa oceanica Zone; Late Pleistocene.

Sample 85-5-2 $(6-7 \mathrm{~cm})$ :

No calcareous nannofossils.

\section{HOLE 86}

Samples: 86-1-1 (7-8 cm); 86-1-2 (6-7 cm); 86-1-3 (6-7 cm): These samples contain Gephyrocapsa oceanica, $G$. aperta, G. caribbeanica, Rhabdosphaera stylifera, and Scapholithus fossilis.

Age: Gephyrocapsa oceanica Zone; Late Pleistocene.

Sample 86-2-1 $(57-58 \mathrm{~cm})$ :

Moderately good assemblage with Gephyrocapsa caribbeanica, both elliptical and varieties of Pseudoemiliania lacunosa, Helicopontosphaera kamptneri, and Rhabdosphaera clavigera. Age: Gephyrocapsa caribbeanica Zone; Early Pleistocene.

Sample 86-3-1 $(7-8 \mathrm{~cm})$ :

This sample contains Gephyrocapsa caribbeanica, $C y$ clococcolithina leptopora, Pseudoemiliania lacunosa, Discoaster brouweri, and D. pentaradiatus.

Age: Probably Discoaster pentaradiatus Zone or Discoaster surculus Zone; Late Pliocene.

Sample 86-3-2 $(6-7 \mathrm{~cm})$ :

This sample contains the same assemblage as that immediately above, plus Sphenolithus abies.

Age: Early Discoaster surculus Zone; Late Pliocene.

Sample 86-3-3 $(7-8 \mathrm{~cm})$ :

Assemblage like that above, but badly corroded.

Samples: 86-3-4 $(5-6 \mathrm{~cm}) ; 86-3-5(9-10 \mathrm{~cm}) ;$ 86-3-6 (7-8 $\mathrm{cm})$ :

Moderately good assemblages like Sample 86-3-2 (6-7 $\mathrm{cm})$, but with Discoaster extensus.

Age: Early Discoaster surculus Zone; Late Pliocene.

Samples 86-4-1 $(18-19 \mathrm{~cm})$; 86-4-2 $(6-7 \mathrm{~cm})$ :

Same assemblage as in Sample 86-3-6 (7-8 cm), but with Discoaster asymmetricus and Reticulofenestra pseudoumbilica.

Age: Reticulofenestra pseudoumbilica Zone; Early Pliocene.

Samples: $86-4-3(6-7 \mathrm{~cm}) ; 86-4-4(6-7 \mathrm{~cm}) ; 86-4-5(9-10$ $\mathrm{cm})$ :

As above but with Discoaster variabilis added. Age: Reticulofenestra pseudoumbilica Zone; Early Pliocene. 
Samples: 86-5-1 $(87-88 \mathrm{~cm}) ; 86-5-2(6-7 \mathrm{~cm})$ :

Assemblage with Discoaster nephados, D. saundersi, Coccolithus pelagicus, Sphenolithus distentus, S. predistentus, and Triquetrorhabdulus carinatus.

Age: Probably Sphenolithus distentus Zone; Middle or Late Oligocene. The overlap of Sphenolithus predistentus and Triquetrorhabdulus carinatus should not occur and may be an indication of mixing of material of different ages.

Samples: $86-5-3(11-12 \mathrm{~cm})$; 86-5-4 $(4-5 \mathrm{~cm})$ :

Sphenolithus predistentus is common in these samples and no Triquetrorhabdulus carinatus occur.

Age: Sphenolithus predistentus Zone; Middle Oligocene.

Samples: 86-7-2 (6-7 cm); 86-7-3 (6-7 cm); 86-7-4 (4-5 cm); $86-7-5(6-7 \mathrm{~cm})$ :

Generally poor assemblages with Coccolithus pelagicus, Chiasmolithus sp., Braarudosphaera bigelowi, B. undata, and Sphenolithus radians.

Age: Uncertain, possibly Lower Eocene.

Sample 86-8-1 (101-102):

No calcareous nannofossils.

Sample 86-8-2 $(7-8 \mathrm{~cm})$ :

Moderately good assemblage with $Z$ Zygodiscus adamas, Chiasmolithus bidens, Coccolithus cavus, Heliolithus kleinpelli, Fasciculithus tympaniformis, and Sphenolithus anarrhopus.

Age: Heliolithus kleinpelli Zone; Middle Paleocene.

Samples: 86-8-3 $(6-7 \mathrm{~cm}) ; 86-8-4(6-7 \mathrm{~cm}) ; 86-9-1(8-10$ $\mathrm{cm})$; 86-9-2 $(6-7 \mathrm{~cm})$ :

Very poor assemblages; resembling that in Sample 868-2 $(7-8 \mathrm{~cm})$, but lacking Heliolithus kleinpelli.

Age: Probably Fasciculithus tympaniformis Zone; Early or Middle Paleocene.

\section{HOLE 87}

Sample 87-1-1 $(6-7 \mathrm{~cm})$ :

Moderately good assemblage with Gephyrocapsa oceanica, Cyclococcolithina leptopora, Cyclolithella annula, Helicopontosphaera kamptneri, and Scapholithus fossilis.

Age: Gephyrocapsa oceanica Zone; Late Pleistocene.

Samples: 87-1-1 $(116-117 \mathrm{~cm})$; 87-1-2 $(6-7 \mathrm{~cm})$ :

No calcareous nannofossils.

\section{HOLE 88}

Samples: 88-1-2 (6-7 cm); 88-1-2 (145-146 cm); 88-1-3 (6-7 $\mathrm{cm})$ :

Good assemblage with Gephyrocapsa oceanica, Helicopontosphaera kamptneri, Scapholithus fossilis, Rhabdosphaera clavigera, $R$. stylifera.

Age: Gephyrocapsa oceanica Zone; Late Pleistocene.

Sample 88-1-4 $(6-7 \mathrm{~cm})$ :

Same assemblage as above but with Gephyrocapsa caribbeanica and G. aperta.

Age: Gephyrocapsa oceanica Zone; Late Pleistocene.

Samples: 88-2-1 $(6-7 \mathrm{~cm})$; 88-2-2 $(6-7 \mathrm{~cm})$; 88-2-3 (12-13 $\mathrm{cm}) ; 88-2-4(8-9 \mathrm{~cm}) ; 88-2-5(3-4 \mathrm{~cm})$ :
Good assemblages with Gephyrocapsa oceanica, $G$. aperta, G. caribbeanica, Helicopontosphaera kamptneri, and Pseudoemiliania lacunosa.

Age: Early Gephyrocapsa oceanica Zone; Late Pleistocene.

Samples: 88-3-3 $(69-70 \mathrm{~cm})$; 88-3-4 $(5-6 \mathrm{~cm})$ :

Good assemblage with Discoaster brouweri rutellus, $D$. brouweri tridenus, and $D$. brouweri tamalis accompanying a flood of Pseudoemiliania lacunosa.

Age: Discoaster brouweri Zone; Late Pliocene.

Samples: 88-3-5 $(5-6 \mathrm{~cm})$; 88-3-6 $(27-28 \mathrm{~cm})$ :

Same assemblage as in the upper part of this barrel, but with Discoaster pentaradiatus.

Age: Discoaster pentaradiatus Zone; Late Pliocene.

Samples: 88-4-1 (6-7 cm); 88-4-2 (125-126 cm); 88-4-3 $(19-20 \mathrm{~cm}) ; 88-4-4(48-49 \mathrm{~cm})$ :

Assemblages with Discoaster brouweri, $D$. pentaradiatus, D. surculus (rare), and Pseudoemiliania lacunosa.

Age: Discoaster surculus Zone; Late Pliocene.

Samples: 88-4-5 (13-14 cm); 88-5-1 (2-3 cm); 88-5-6 (28-29 $\mathrm{cm})$; 88-5-6 (18-19 cm):

Assemblages with Discoaster brouweri, D. pentaradiatus, D. surculus, Reticulofenestra pseudoumbilica. Age: Reticulofenestra pseudoumbilica Zone; Early Pliocene.

\section{HOLE 89}

Samples: 89-1-1 (100-101 cm); 89-1-2 $(19-20 \mathrm{~cm})$ :

Assemblages of very small coccoliths, including $\mathrm{Ge}$ phyrocapsa oceanica, G. aperta, G. caribbeanica, and $C y$ clococcolithina leptopora.

Age: Gephyrocapsa oceanica Zone; Late Pleistocene.

Sample 89-1-3 (12-13 cm):

Very poor assemblage with a few large specimens of Gephyrocapsa oceanica.

Age Gephyrocapsa oceanica Zone; Late Pleistocene.

Sample 89-2-1 (122-123 cm):

Good assemblage with many Gephyrocapsa caribbeanica and Pseudoemiliania lacunosa, some Cycloccolithina leptopora and Helicopontosphaera kamptneri. Age: Gephyrocapsa caribbeanica Zone; Early Pleistocene.

Samples: 89-3-1 $(4-5 \mathrm{~cm})$; 89-3-2 $(6-7 \mathrm{~cm})$ :

Moderately good assemblages with Gephyrocapsa caribbeanica, Pseudoemiliania lacunosa, Rhabdosphaera clavigera, and Helicopontosphaera kamptneri.

Age: Gephyrocapsa caribbeanica Zone; Early Pleistocene.

Sample 89-3-3 (10-11 cm):

Same assemblage as above, but with fragments of Discoaster brouweri.

Age: Earliest Gephyrocapsa caribbeanica Zone or latest Discoaster brouweri Zone; Earliest Pleistocene or latest Pliocene.

Sample 89-3-4 $(6-7 \mathrm{~cm})$ :

Gephyrocapsa caribbeanica, Pseudoemiliania lacunosa, Discoaster brouweri, and $D$. pentaradiatus.

Age: Discoaster pentaradiatus Zone, Late Pliocene. 
Sample 89-4-1 $(6-7 \mathrm{~cm})$ :

Good assemblage with Gephyrocapsa caribbeanica, Reticulofenestra pseudoumbilica, Sphenolithus abies, Discoaster brouweri, $D$. pentaradiatus, $D$. cf. surculus, $D$. cf. extensus.

Age: Probably Discoaster surculus Zone; Late Pliocene.

Sample 89-4-2 $(6-7 \mathrm{~cm})$ :

Same species as above are present, but the assemblage is very poor.

Sample 89-4-3 $(7-8 \mathrm{~cm})$ :

Same assemblage as in Sample 89-4-1 (6-7 cm), but with typical specimens of Discoaster surculus.

Age: Discoaster surculus Zone; Late Pliocene.

Sample 89-4-4 $(6-7 \mathrm{~cm})$ :

Same assemblage as in Sample 89-4-3 $(7-8 \mathrm{~cm})$, but with a reworked specimen of Discoaster barbadiensis.

Sample 89-4-5 $(24-25 \mathrm{~cm})$ :

Good assemblage with Reticulofenestra pseudoumbilica, Sphenolithus abies, Discoaster brouweri, D. pentaradiatus, D. surculus, Ceratolithus tricorniculatus, and C. rugosus.

Age: Ceratolithus rugosus Zone; Early Pliocene.

Sample 89-4-6 $(7-8 \mathrm{~cm})$ :

Same assemblage as in Sample 89-4-5 (24-25 cm), but with Discoaster cf. quinqueramus.

Age: Ceratolithus rugosus Zone; Early Pliocene.

Sample 89-5-1 (106-107 cm):

Very sandy sample with a few specimens of Reticulofenestra pseudoumbilica.

Age: Miocene or Pliocene.

Sample 89-6-1 $(84-85 \mathrm{~cm})$ :

Very poor sample with only a few corroded unidentifiable coccoliths.

Age: Uncertain.

Sample 89-6-3 $(6-7 \mathrm{~cm})$ :

Poor assemblage with Discoaster brouweri, D. quinqueramus, and $D$. cf. neohamatus.

Age: Probably Discoaster quinqueramus Zone; Late Miocene.

Sample: $89-6-4(11-12 \mathrm{~cm})$ :

Poor assemblage with Discoaster brouweri, $D$. pentaradiatus, D. quinqueramus, and reworked specimens of Micula staurophora.

Age: Discoaster quinqueramus Zone; Late Miocene

Sample 89-6-5 $(11-12 \mathrm{~cm})$ :

Poor assemblage with Discoaster brouweri, D. pentaradiatus, $D$. quinqueramus, $D$. cf. neohamatus, and reworked specimens of Microrhabdulus decoratus and Micula staurophora.

Age: Discoaster quinqueramus Zone; Late Miocene.

\section{HOLE 90}

Sample 90-1-1 (6-7 cm):

Good assemblage with Gephyrocapsa oceanica, G. caribbeanica, Helicopontosphaera kamptneri, Discolithina sp., Rhabdosphaera stylifera.

Age: Gephyrocapsa oceanica Zone; Late Pleistocene.
Sample 90-1-2 (6-7 cm):

Sandy sample with very poor assemblage like that above.

Samples: 90-1-3 (6-7 cm); 90-1-4 (6-7 cm); 90-1-5 (6-7 cm); 90-1-6 $(6-7 \mathrm{~cm})$ :

Good assemblages with Gephyrocapsa oceanica, $G$. aperta, G. caribbeanica, Ceratolithus cristatus, Discolithina phaseola, Helicopontosphaera kamptneri, Cyclococcolithina leptopora, and Rhabdosphaera styifera.

Age: Gephyrocapsa oceanica Zone; Late Pleistocene.

Samples: 90-2-1 (57-58); 90-2-2 (6-7 cm); 90-2-3 (15-16 $\mathrm{cm})$; 90-2-4 $(6-7 \mathrm{~cm})$ :

Same assemblage as in Sample 90-1-6 (6-7 cm), but with Pseudoemiliania lacunosa.

Age: Early Gephyrocapsa oceanica Zone; Late Pleistocene. Samples: 90-3-1 (60-61 cm); 90-3-2 (6-7 cm); 90-3-3 (14-15 $\mathrm{cm})$ :

Assemblages with Discoaster brouweri rutellus, D. brouweri tridenus, D. brouweri tamalis, and D. pentaradiatus.

Age: Discoaster pentaradiatus Zone; Late Pliocene.

Samples: 90-4-1 (86-87 cm); 90-4-2 (6-7 cm); 90-4-3 (13-14 $\mathrm{cm})$ :

Assemblages with Discoaster brouweri, D. pentaradiatus, D. surculus, and Sphenolithus abies.

Age: Early Discoaster surculus Zone; Late Pliocene.

Sample 90-5-1 (6-7 cm):

Very poor assemblage with Discoaster brouweri, D. pentaradiatus, D. surculus.

Age: Late Miocene or Pliocene.

Sample 90-5-2 $(7-8 \mathrm{~cm})$

Very poor assemblage with only a few unidentifiable coccoliths.

Age: Uncertain.

Sample 90-5-3 (3-4 cm):

Very poor assemblage, but with Discoaster quinqueramus.

Age: Discoaster quinqueramus Zone; Late Miocene.

Samples: 90-5-4 (6-7 cm); 90-6-1 (19-20 cm); 90-6-2 (6-7

$\mathrm{cm})$ :

No calcareous nannofossils.

Samples 90-7-1 (32-33 cm); 90-7-2 (6-7 cm); 90-7-3 (6-7 $\mathrm{cm})$ :

Very poor assemblages with Discoaster brouweri rutellus, $D$. brouweri tridenus, $D$. brouweri triradiatus, $D$. expansus, and $D$. quinqueramus.

Age: Discoaster quinqueramus Zone; Late Miocene.

Samples: 90-9-1 (26-27 cm); 90-10-1 (3-4 cm):

No calcareous nannofossils.

Sample 90-11-2 (11-12 cm):

Sample almost barren, containing only a few coccolith and asterolith fragments.

Sample 90-11-3 $(6-7 \mathrm{~cm})$ :

Very poor sample containing some heavily calcified asterolith fragments. Apparently only five- and six-rayed asteroliths are present.

Age: Probably Middle or Late Miocene. 
Samples: $90-11-4(6-7 \mathrm{~cm}) ; 90-11-5(6-7 \mathrm{~cm}) ; 90-11-6(6-7$ $\mathrm{cm}) ; 90-13-1(6-7 \mathrm{~cm}) ; 90-13-2(6-7 \mathrm{~cm}) ; 90-13-3(19-20$ $\mathrm{cm})$ :

No calcareous nannofossils.

\section{HOLE 91}

Sample 91-1-1 (90-91 cm): No calcareous nannofossils.

Sample 91-1-2 (6-7 cm):

Very poor assemblage with Gephyrocapsa oceanica, $G$. caribbeanica, and Helicopontosphaera kamptneri.

Age: Gephyrocapsa caribbeanica Zone; Late Pleistocene.

Samples: 91-1-3 (3-4 cm); 91-1-4 (14-15 cm); 91-1-5 (6-7 $\mathrm{cm})$; $91-1-6(6-7 \mathrm{~cm}) ; 91-2-1(122-123 \mathrm{~cm})$ :

No calcareous nannofossils.

Samples: 91-2-2 (6-7 cm); 91-2-3 (6-7 cm); 91-2-4 (5-6 cm): Very poor samples with a few specimens of $\mathrm{Ge}$ phyrocapsa oceanica, G. caribbeanica, and $G$. aperta.

Age: Gephyrocapsa caribbeanica Zone; Late Pleistocene.

Sample 91-2-5 $(6-7 \mathrm{~cm})$ :

No calcareous nannofossils.

Sample 91-3-1 (6-7 cm):

Very poor sample with Helicopontosphaera kamptneri and reworked Mesozoic Watznaueria barnesae.

Samples: 91-3-2 (4-5 cm); 91-3-6 $(6-7 \mathrm{~cm})$ :

No calcareous nannofossils.

Sample 91-4-1 (6-7 cm):

A few reworked Cretaceous coccoliths occur: Eiffellithus turriseiffeli, Watznaueria barnesae, Vekshinella sp., Micula staurophora.

Samples: 91-4-2 (6-7 cm); 91-4-3 $(6-7 \mathrm{~cm})$ :

Very poor samples with only a few specimens of reworked Mesozoic Watznaueria barnesae.

Samples: 91-4-4 (6-7 cm);91-4-5 (6-7 cm);91-4-6 (6-7 cm): No calcareous nannofossils.

Sample 91-5-1 (5-6 cm):

Very poor assemblage with Gephyrocapsa oceanica and G. aperta.

Age: Gephyrocapsa oceanica Zone; Late Pleistocene.

Sample 91-5-2 (5-6 cm):

Very poor assemblage containing Gephyrocapsa aperta, G. caribbeanica, and Helicopontosphaera kamptneri.

Age: Probably Gephyrocapsa oceanica Zone; Late Pleistocene.

Samples: $91-5-3(6-7 \mathrm{~cm}) ; 91-5-4(6-7 \mathrm{~cm}) ; 91-5-5(6-7 \mathrm{~cm})$ : No calcareous nannofossils.

Sample 91-5-6 (5-6 cm):

Poor assemblage with Gephyrocapsa oceanica, $G$. aperta, G. caribbeanica, and reworked Cretaceous Watznaueria barnesae.

Age: Gephyrocapsa oceanica Zone; Late Pleistocene.

Sample 91-6-1 (6-7 cm):

Moderately good assemblage with Gephyrocapsa oceanica, G. aperta, G. caribbeanica, Cyclococcolithina leptopora, Scapholithus fossilis, and Rhabdosphaera stylifera. Age: Gephyrocapsa oceanica Zone; Late Pleistocene.
Sample 91-6-2 (6-7 cm):

Poor assemblage of reworked Cretaceous coccoliths: Arkhangelskiella cymbiformis, Eiffellithus turriseiffeli, Prediscosphaera cretacea, Watznaueria barnesae, Micula staurophora.

Sample 91-6-3 (6-7 cm):

Very poor assemblage with only a few rims of Cretaceous coccoliths.

Sample 91-6-4 (5-6 cm):

No calcareous nannofossils.

Sample 91-6-5 $(5-6 \mathrm{~cm})$ :

Very poor assemblage with Gephyrocapsa oceanica, and some reworked Cretaceous specimens of Watznaueria barnesae, Micula staurophora, and Cribrosphaera ehrenbergi. Age: Gephyrocapsa oceanica Zone; Late Pleistocene.

Samples: 91-7-1 (45-46 cm); 91-7-2 (? cm); 91-7-3 (6-7 $\mathrm{cm})$ :

No calcareous nannofossils.

Sample 91-7-4 (6-7 cm):

Very poor assemblage with Gephyrocapsa oceanica, $C y$ clococcolithina leptopora, and reworked Watznaueria barnesae.

Age: Gephyrocapsa oceanica Zone; Late Pleistocene.

Sample 91-7-5 $(6-7 \mathrm{~cm})$ :

No calcareous nannofossils.

Samples: 91-8-1 (23-24 cm); 91-8-2 (6-7 cm):

Very poor assemblages with Gephyrocapsa caribbeanica, Pseudoemiliania lacunosa, and Discoaster brouweri.

Age: Discoaster brouweri Zone; Late Pleiocene.

Samples: 91-9-2 (7-8 cm); 91-9-3 (6-7 cm); 91-9-4 (6-7 cm): No calcareous nannofossils.

Sample 91-9-5 (7-8 cm):

Moderately good assemblage with Pseudoemiliania lacunosa, Gephyrocapsa caribbeanica, Discoaster brouweri rutellus, D. brouweri tridenus, D. brouweri tamalis, $D$. pentaradiatus, and Ceratolithus cristatus.

Age: Discoaster pentaradiatus Zone; Late Pliocene.

Sample 91-9-6 (12-13 cm):

Very poor sample with only a few specimens of Pseudoemiliania lacunosa.

Sample 91-10-2 $(6-7 \mathrm{~cm})$ :

Very poor assemblage with Discoaster brouweri and $D$. pentaradiatus.

Age: Discoaster pentaradiatus Zone; Late Pliocene.

Samples: 91-10-3 (6-7 cm); 91-10-4 (6-7 cm):

No calcareous nannofossils.

Sample 91-11-2 (6-7 cm):

Very poor, with only a few coccolith rims present.

Samples: $91-11-3(4-5 \mathrm{~cm})$; 91-11-4 (6-7 cm); 91-12-2 (6-7 $\mathrm{cm})$; $91-12-3(8-9 \mathrm{~cm}) ; 91-13-1(58-59 \mathrm{~cm}) ; 91-13-2(6-7$ $\mathrm{cm})$; $91-13-3(9-10 \mathrm{~cm})$; $91-14-2(6-7 \mathrm{~cm} ; 19-15-1(6-7 \mathrm{~cm})$ : No calcareous nannofossils.

Samples: 91-15-2 (6-7 cm); 91-15-3 (6-7 cm):

Poor assemblage with Discoaster brouweri, D. extensus, D. kugleri, D. bollii.

Age: Discoaster kugleri Zone; Middle Miocene. 
Sample 91-16-1 (3-4 cm):

No calcareous nannofossils.

Sample 91-16-2 (6-7 cm):

Poor assemblage with Discoaster brouweri, D. bollii, $D$. kugleri, and Catinaster calyculus.

Age: Discoaster kugleri Zone, Middle Miocene.

Samples: $91-16-3(6-7 \mathrm{~cm})$; 91-18-6 $(73-74 \mathrm{~cm})$ : 91-20-2

$(6-7 \mathrm{~cm})$; $91-20-6(7-8 \mathrm{~cm}) ; 91-22-1(6-7 \mathrm{~cm})$ :

No calcareous nannofossils.

Sample 91-22-2 $(5-6 \mathrm{~cm})$ :

Very poor assemblage with Coccolithus cf. pelagicus, and asterolith fragments.

Age: Uncertain.

Samples: 91-25-1 $(89-90 \mathrm{~cm})$; 91-25-2 $(6-7 \mathrm{~cm})$; 91-25-3

(13-14 cm); 91-25-4 (12-13 cm):

No calcareous nannofossils.

\section{HOLE 92}

Sample 92-2-1 $(91-92 \mathrm{~cm})$ :

No calcareous nannofossils.

Sample 92-2-2 $(6-7 \mathrm{~cm})$ :

Very poor assemblage of reworked Cretaceous coccoliths, with Watznaueria barnesae and Micula staurophora.

Sample 92-2-3 $(6-7 \mathrm{~cm})$ :

Very poor assemblage with Gephyrocapsa caribbeanica, and reworked Cretaceous calcareous nannofossils: $M i$ crorhabdulus decoratus, Arkhangelskiella sp., Micula staurophora, Prediscosphaera cretacea.

Age: Probably Gephyrocapsa caribbeanica Zone; Early Pleistocene.

Sample 92-2-4 $(6-7 \mathrm{~cm})$ :

Few Cretaceous calcareous nannofossils present: $M i-$ crorhabdulus decoratus, Micula staurophora.

Sample 92-2-5 $(6-7 \mathrm{~cm})$ :

Poor assemblage of reworked Cretaceous calcareous nannofossils, with Watznaueria barnease, Arkhangelskiella cymbiformis, and Prediscophaera cretacea.

Sample 92-3-1 (101-102 cm):

Very poor assemblage with a few specimens of $\mathrm{Ge}$ phyrocapsa caribbeanica and $G$. aperta.

Age: Probably Gephyrocapsa caribbeanica Zone: Early Pleistocene.

Samples: 92-3-2 (6-7 cm); 92-3-3 $(6-7 \mathrm{~cm})$; 92-3-4 (6-7 cm); 92-3-5 (6-7 cm); 92-4-2 (6-7 cm); 92-4-3 $(6-7 \mathrm{~cm})$ :

No calcareous nannofossils.

Sample 92-4-4 $(6-7 \mathrm{~cm})$ :

Very poor sample with a few specimens of Watznaueria barnesae and Gephyrocapsa sp.

Sample 92-4-5 $(9-10 \mathrm{~cm})$ :

No calcareous nannofossils.

Sample 92-5-1 $(6-7 \mathrm{~cm})$ :

Very poor assemblage with Gephyrocapsa sp. and reworked Cretaceous coccoliths: Arkhangelskiella cymbiformis, Watznaueria barnesae, and Prediscosphaera cretacea.
Sample: $92-5-2(6-7 \mathrm{~cm})$ :

Very rare, poorly preserved specimens of Gephyrocapsa cf. caribbeanica.

Age: ? Gephyrocapsa caribbeanica Zone; ? Early Pleistocene.

Samples:92-5-3, $(6-7 \mathrm{~cm}) ; 92-5-4,(6-7 \mathrm{~cm})$ :

Only a few coccolith remnants are present.

Sample 92-5-5, (6-7 cm):

Micula staurophora and Watznaueria barnesae from the Cretaceous occur with rare, poor specimens of $G e-$ phyrocapsa cf. caribbeanica.

Age: ? Gephyrocapsa caribbeanica Zone; ? Early Pleistocene.

Sample: $92-5-6(8-9 \mathrm{~cm})$ :

Only a few reworked Cretaceous coccoliths are present: Prediscosphaera cretacea, Arkhangelskiella parca, Eiffellithus turriseiffeli, and Watznaueria barnesae.

Samples: 92-6-2 (6-7 cm); 92-7-1 (126-127 cm); 92:8-1 $(34-35 \mathrm{~cm})$; $92-11-2(6-7 \mathrm{~cm})$ :

No calcareous nannofossils.

\section{HOLE 93}

Sample 93-1-1 (133-134 cm):

Mixed assemblage with Gephyrocapsa oceanica, $G$. caribbeanica, Cyclococcolithina leptopora, Helicopontosphaera kamptneri, and Discoaster brouweri.

Age: Mixed Late Pleistocene and Late Pliocene.

Sample 93-1-2 $(6-7 \mathrm{~cm})$ :

Good assemblage with Sphenolithus abies, Discoaster pentaradiatus, D. brouweri, D. extensus, Helicopontosphaera kamptneri, and Coccolithus pelagicus.

Age: Probably Discoaster surculus Zone; Late Pliocene.

\section{HOLE 94}

Samples: 94-1-1 (103-104 cm); 94-1-2 (6-7 cm):

Good assemblage with Gephyrocapsa oceanica, $G$. caribbeanica, G. aperta, Helicopontosphaera kamptneri, and Cyclococcolithina leptopora.

Age: Gephyrocapsa oceanica Zone; Late Pleistocene.

Samples: 94-2-1 (6-7 cm); 94-2-2 (6-7 cm):

Good assemblage with Gephyrocapsa caribbeanica, $G$. aperta, Pseudoemiliania lacunosa, Helicopontosphaera kamptneri, and Cyclococcolithina leptopora.

Age: Gephyrocapsa caribbeanica Zone; Early Pleistocene.

Samples: 94-2-3 (6-7 cm); 94-2-4 (6-7 cm); 94-2-5 (6-7 cm); 94-2-6 $(6-7 \mathrm{~cm})$ :

Good assemblage with Gephyrocapsa caribbeanica, Pseudoemiliania lacunosa, Discoaster brouweri, and $D$. pentaradiatus.

Age: Discoaster pentaradiatus Zone; Late Pliocene.

Samples: 94-3-1 (33-34 cm); 94-3-2 $(6-7 \mathrm{~cm})$ :

Good assemblage with Gephyrocapsa caribbeanica, Pseudoemiliania lacunosa, Discoaster brouweri, D. pentaradiatus, D. cf. surculus, D. asymmetricus, and Sphenolithus abies.

Age: Early Discoaster surculus Zone; Late Pliocene. 
Sample 94-3-3 (6-7 cm):

Poor assemblage, but with the same species as immediately above.

Sample 94-3-4 (17-18 cm):

Moderately good assemblage like that of Sample 94-3-2 $(6-7 \mathrm{~cm})$

Samples; 94-3-5 (6-7 cm); 94-3-6 (6-7 cm):

Assemblages like in Sample 94-3-2 (6-7 cm), but with typical Discoaster surculus present.

Age: Early Discoaster surculus Zone; Late Pliocene.

Sample 94-4-1 (6-7 cm):

Good assemblage with Discoaster brouweri, D. surculus, $D$. pentaradiatus, $D$. asymmetricus, Reticulofenestra pseudoumbilica, and Ceratolithus rugosus.

Age: Reticulofenestra pseudoumbilica Zone; Early Pliocene.

Sample 94-4-2 (6-7 cm):

Good assemblage with Discoaster brouweri, D. surculus, D. pentaradiatus, D. perplexus, D. asymmetricus, Reticulofenestra pseudoumbilica, Ceratolithus tricorniculus.

Age: Discoaster asymmetricus Zone; Early Pliocene.

Sample 94-4-3 (12-13 cm):

Assemblage as immediately above, but apparently lacking Discoaster asymmetricus.

Age: Ceratolithus rugosus Zone; Early Pliocene.

Samples: 94-4-4 (6-7 cm); 94-4-5 (6-7 cm):

Assemblages with the same species as in Sample 94-4-3 $(12-13 \mathrm{~cm})$, but dominated by a flood of minute coccoliths. Age: Ceratolithus rugosus Zone; Early Pliocene.

Samples: 94-4-6 (6-7 cm); 94-5-1 (14-15 cm); 94-5-2 (6-7 $\mathrm{cm})$; $94-5-3(6-7 \mathrm{~cm})$; 94-5-4 (14-15 cm):

Moderately good samples with Discoaster brouweri, $D$. pentaradiatus, D. surculus, Reticulofenestra pseudoumbilica, Ceratolithus tricorniculatus and $C$. rugosus.

Age: Ceratolithus rugosus Zone; Early Pliocene.

Samples: 94-5-5 (6-7 cm); 94-6-1 (6-7 cm); 94-6-2 (4-5 cm); 94-6-3 (6-7 cm); 94-6-4 (13-14 cm); 94-6-5 (4-5 cm); 94-6-6 $(6-7 \mathrm{~cm}) ; 94-7-1(108-109 \mathrm{~cm}) ; 94-7-2(6-7 \mathrm{~cm}) ; 94-7-3(6-7$ $\mathrm{cm})$; $94-7-4(6-7 \mathrm{~cm})$ :

Poor to very poor assemblages with few asteroliths and very rare ceratoliths; species mostly as in Sample 94-5-4 (14-15 cm)

Age: Probably Ceratolithus rugosus Zone or Ceratolithus tricorniculatus Zone; Early Pliocene or Late Miocene.

Sample 94-7-5 (6-7 cm):

Moderately good assemblage with Discoaster brouweri, D. surculus, and D. quinqueramus.

Age: Discoaster quinqueramus Zone; Late Miocene.

Sample 94-8-1 (111-112 cm); 94-8-2 (6-7 cm):

Good assemblages with abundant Discoaster quinqueramus.

Age: Discoaster quinqueramus Zone; Late Miocene.

Samples: 94-8-3 (6-7 cm); 94-8-4 (6-7 cm); 94-8-5 (6-7 cm):

Good assemblages with Discoaster quinqueramus, $D$. neohamatus, D. brouweri, D. extensus, D. surculus, and $D$. perclarus.

Age: Discoaster quinqueramus Zone; Late Miocene.
Sample 94-9-1 $(6-7 \mathrm{~cm})$ :

Poor assemblage with a few corroded coccoliths and asteroliths.

Samples: 94-9-2 (6-7 cm); 94-9-3 (6-7 cm); 94-9-4 (6-7 cm): Good assemblages with Discoaster aulakos, D. dilatus, D. nephados, D. cf. exilis, D. extensus, D. variabilis, Discolithina anisotrema, and Sphenolithus heteromorphus.

Age: Sphenolithus heteromorphus Zone; Middle Miocene. Sample 94-9-6 $(6-7 \mathrm{~cm})$ :

Poor assemblage containing Helicopontosphaera parallela, Sphenolithus ciperoensis, and a few nondescript asteroliths.

Age: Probably Discoaster druggi Zone; Early Miocene.

Samples: $94-10-1(69-70 \mathrm{~cm}) ; 94-10-2$ (6-7 cm); 94-10-3 $(6-7 \mathrm{~cm})$ :

Good assemblages with Triquetrorhabdulus carinatus, Coronocyclus nitescens, Cyclococcolithina floridana, Discoaster aulakos, D. deflandrei, D. adamanteus, D. saundersi, and $D$. extensus.

Age: Probably Triquetrorhabdulus carinatus Zone; Late Oligocene or Early Miocene.

Sample 94-11-1 (103-104 cm):

Assemblage similar to that above, but lacking Triquetrorhabulus carinatus, and with Sphenolithus distentus and Reticulofenestra bisecta.

Age: Sphenolithus distentus Zone; Middle or Late Oligocene.

Samples: $94-11-2(6-7 \mathrm{~cm}) ; 94-11-3(14-15 \mathrm{~cm}) ; 94-11-4$ $(6-7 \mathrm{~cm})$ :

Good assemblage with Reticulofenestra bisecta, Discoaster trinidadensis, D. saundersi, D. adamanteus, $C y$ clococcolithina floridana, Sphenolithus distentus and $S$. predistentus.

Age: Sphenolithus predistentus Zone; Middle Oligocene.

Samples: $94-11-5$ (6-7 cm); 94-11-6 (6-7 cm); 94-12-1 (19-20 cm); 94-12-2 (6-7 cm); 94-12-3 (6-7 cm); 94-12-4 $(6-7 \mathrm{~cm})$; $94-13-2(22-23 \mathrm{~cm}) ; 94-13-3(6-7 \mathrm{~cm})$ :

Good assemblages with the same species as in Sample 94-11-4, except that Sphenolithus distentus is absent, and rare specimens of Discoaster tani ornatus occur.

Age: Sphenolithus predistentus Zone; Middle Oligocene.

Samples: $94-14-1(119-120 \mathrm{~cm})$ : 94-14-2 (5-6 cm); 94-14-3

(6-7 cm); 94-14-4 (4-5 cm); 94-15-4 (39-40 cm):

Moderately good assemblages with Cyclococcolithina formosa, Reticulofenestra umbilica, Discoaster barbadiensis, D. saipanensis, D. tani ornatus, and Ericsonia subdisticha.

Age: Probably Isthmolithus recurvus Zone or Sphenolithus pseudoradians Zone; Late Eocene.

Sample 94-16-1 (11-12 cm):

Very poor assemblage, lacking Discoaster barbadiensis. Samples: 94-16-2 (6-7 cm); 94-16-3 (6-7 cm); 94-17-1 (16-17 cm); 94-17-2 (12-13 cm); 94-17-3 (7-8 cm):

Moderately good assemblages, with species as in Sample $94-15-4(39-40 \mathrm{~cm})$

Sample 94-17-4 (6-7 cm):

Very poor sample with only a few small corroded coccoliths. 
Samples: 94-18-1 (6-7 cm); 94-18-2 (6-7 cm):

Moderately good assemblage with Reticulofenestra umbilica, Discoaster barbadiensis, D. saipanensis, Braarudosphaera undata, and $B$. discula.

Age: Late Eocene.

Sample 94-18-3 (6-7 cm):

Poor assemblage with small coccoliths.

Samples: 94-18-4 (6-7 cm); 94-19-1 (7-8 cm); 94-19-2 (5-6 $\mathrm{cm})$; 94-19-3 (5-6 cm); 94-19-4 (6-7 cm); 94-19-5 (5-6 cm); 94-20-1 (6-7 cm); 94-20-2 (6-7 cm); 94-20-3 (7-8 cm); 94-20-4 (6-7 cm):

Generally poor samples with species as in Sample 94$18-2(6-7 \mathrm{~cm})$.

Age: Probably Late Eocene.

Sample 94-21-2 $(6-7 \mathrm{~cm})$ :

Moderately good sample with Zygrhablithus bijugatus, Triquetrorhabdulus inversus, Micrantholithus procerus, Braarudosphaera discula, Discaoster barbadiensis, and D. saipanensis.

Age: Probably Middle Eocene.

Samples: $94-22-1(15-16 \mathrm{~cm})$; 94-22-2 $(6-7 \mathrm{~cm})$; 94-22-3 $(6-7 \mathrm{~cm})$ :

Moderately good assemblages with Discoaster barbadiensis, D. saipanensis, Reticulofenestra umbilica, Zygrhablithus bijugatus, Micrantholithus procerus, and Heliorthus concinnus.

Age: Probably Middle Eocene.

Samples: $94-22-4(6-7 \mathrm{~cm}) ; 94-23-1(89-90 \mathrm{~cm}) ; 94-23-2$ $(6-7 \mathrm{~cm})$; 94-24-1 (115-116 cm); 94-24-2 (6-7 cm); 94-24-3 $(7-8 \mathrm{~cm})$ :

Mediocre to poor samples with the same assemblage as Sample 94-22-3 $(6-7 \mathrm{~cm})$.

Sample 94-24-4 $(6-7 \mathrm{~cm})$ :

Good assemblage with Discoaster barbadiensis, D. deflandrei, Coccolithus eopelagicus, Triquetrorhabdulus inversus, Micrantholithus procerus, Campylosphaera dela, Chiasmolithus grandis.

Age: Middle Eocene.

Samples: 94-25-1 $(22-23 \mathrm{~cm})$; 94-25-2 $(6-7 \mathrm{~cm})$ :

Poor assemblages, but with the same species as immediately above.

Sample 94-25-3 $(7-8 \mathrm{~cm})$ :

Good assemblage like that of Sample 94-24-4 $(6-7 \mathrm{~cm})$. Age: Middle Eocene

Samples: 94-26-1 (103-104 cm); 94-26-2 (6-7 cm); 94-26-3 $(6-7 \mathrm{~cm}) ; 94-26-4(6-7 \mathrm{~cm}) ; 94-26-5(6-7 \mathrm{~cm}) ; 94-28-1$ $(80-81 \mathrm{~cm}) ; 94-28-2(6-7 \mathrm{~cm}) ; 94-28-3(6-7 \mathrm{~cm})$ :

Moderately good assemblages with species as in Sample 94-24-4 (6-7 cm).

Age: Middle Eocene.

Samples: 94-28-4 $(6-7 \mathrm{~cm})$; 94-28-5 $(6-7 \mathrm{~cm})$ :

Poor assemblages with species as in Sample 94-24-4 (6-7 cm).

Age: Probably Middle Eocene.

Samples: 94-30-1 $(4-5 \mathrm{~cm})$; $94-30-2(1-2 \mathrm{~cm})$ :

Very poor assemblages with Sphenolithus anarrhopus, Discolithina pectinata, Coccolithus cf. pelagicus, Discoaster barbadiensis, and Lophodolithus reniformis.

Age: Early Eocene.

Sample 94-33-1 (6-7 cm):

Discoaster barbadiensis is the only recognizable calcareous nannofossil.

Sample 94-33-2 $(4-5 \mathrm{~cm})$ :

Very poor assemblage with Discoaster multiradiatus and Neococcolithes protenus.

Age: Discoaster multiradiatus Zone or Marthasterites contortus Zone; Late Paleocene or Early Eocene.

Samples: 94-34-1 (1-2 cm); 94-34-2 (4-5 cm); 94-34-3 (1-2 $\mathrm{cm})$ :

Poor assemblages with Discoaster multiradiatus, Chiasmolithus consuetus, and Fasciculithus tympaniformis. Age: Discoaster multiradiatus Zone; Late Paleocene.

Samples: 94-35-1 (108-109 cm); 94-35-1 $(117-119 \mathrm{~cm})$ :

Mediocre assemblages with Sphenolithus anarrhopus, Discoaster multiradiatus, Fasciculithus tympaniformis, Ericsonia subpertusa, and Zygodiscus adamas.

Age: Discoaster multiradiatus Zone; Late Paleocene.

Sample 94-36-1 $(99-101 \mathrm{~cm})$ :

Very poor assemblage with Ericsonia subpertusa, Coccolithus cavus, and small nondescript coccoliths.

Age: Probably Cruciplacolithus tenuis Zone; Early Paleocene.

Sample 94-38-1 (23-24 cm): No calcareous nannofossils.

\section{HOLE 95}

Samples: 95-1-1 $(14-15 \mathrm{~cm})$; 95-1-5 $(6-7 \mathrm{~cm})$ :

Good assemblages with Gephyrocapsa oceanica, $G$. caribbeanica, G. aperta, and Cyclococcolithina leptopora. Age: Gephyrocapsa oceanica Zone; Late Pleistocene.

Samples: 95-2-1 (6-7 cm); 95-2-2 $(24-25 \mathrm{~cm})$; 95-2-3 (6-7 $\mathrm{cm})$ :

Good assemblages with Triquetrorhabdulus carinatus, Cyclococcolithina floridana, Coccolithus pelagicus, Spenolithus belemnos, Discoaster nephados, D. saundersi, and $D$. deflandrei.

Age: Probably Triquetrorhabdulus carinatus Zone; Late Oligocene or Early Miocene.

Sample 95-2-6 (6-7 cm): $\mathrm{cm}$ )

Poor assemblage with species as in Sample 95-2-3 (6-7

Samples: 95-3-1 $(6-7 \mathrm{~cm})$; 95-3-2 $(28-29 \mathrm{~cm})$; 95-3-3 (5-6 $\mathrm{cm})$; $95-3-4(8-9 \mathrm{~cm})$; 95-3-5 (15-16 cm); 95-3-6 (6-7 cm); 95-4-2 (7-8 cm); 95-4-3 (6-7 cm); 95-4-4 $(6-7 \mathrm{~cm})$ :

Good assemblages with Cyclococcolithina floridana, Coccolithus eopelagicus, Sphenolithus predistentus and $S$. distentus.

Age: Probably Sphenolithus distentus Zone; Middle or Late Oligocene.

Samples: 95-5-1 (2-3 cm); 95-5-2 (13-14 cm); 95-5-3 (6-7 $\mathrm{cm})$; 95-5-4 $(6-7 \mathrm{~cm})$ :

Good assemblages with Cyclococcolithina floridana, Coccolithus eopelagicus and Sphenolithus predistentus. Age: Probably Sphenolithus predistentus Zone; Middle Oligocene. 
Samples: 95-6-1 (6-7 cm); 95-6-2 (6-7 cm); 95-6-3 $(6-7 \mathrm{~cm})$; 95-6-4 (6-7 cm); 95-6-5 (6-7 cm); 95-6-6 (6-7 cm):

Good assemblages with Cyclococcolithina floridana, Coccolithus eopelagicus, Sphenolithus predistentus,

Reticulofenestra umbilica, Coronocyclus nitescens, and Quinquerhabdus colossicus.

Age: Sphenolithus predistentus Zone; Middle Oligocene

Samples: 95-7-1 (5-6 cm); 95-7-2 (6-7 cm); 95-7-3 (6-7 cm); 95-7-4 (4-5 cm); 95-7-5 (5-6 cm); 95-7-6 (6-7 cm); 95-8-1 $(6-7 \mathrm{~cm})$ :

Moderately good assemblages with Coccolithus eopelagicus, Cyclococcolithina floridana, and Discoaster barbadiensis.

Age: Probably Late Eocene.

Samples: $95-8-2(6-7 \mathrm{~cm})$; $95-8-3(6-7 \mathrm{~cm}) ; 95-8-4(6-7 \mathrm{~cm})$; 95-8-5 (6-7 cm); 95-8-6 (6-7 cm):

Moderately good to poor assemblages with Cyclococcolithina floridana, Coccolithus eopelagicus, Chiasmolithus grandis, Triquetrorhabdulus inversus, and Micrantholithus procerus.

Age: Probably Middle Eocene.

Sample 95-10-1 (10-11 cm):

Moderately good assemblage with Ericsonia subpertusa, Fasciculithus tympaniformis, Chiasmolithus bidens, Discoaster multiradiatus, and Braarudosphaera bigelowi. Age: Probably Discoaster multiradiatus Zone; Late Paleocene.

Samples: 95-11-1 (108-110 cm); 95-11-2 (6-7 cm); 95-11-3 (6-7 cm); 95-11-4 (1-2 cm):

Poor assemblages with Fasciculithus tympaniformis, Chiasmolithus danicus, Braarudosphaera discula, $B$. bigelowi, Ericsonia subpertusa, Cruciplacolithus tenuis, Markalius astroporus, Zygodiscus adamus, and Coccolithus cavus.

Age: Fasciculithus tympaniformis Zone; Middle Paleocene.

Samples: 95-12-1 (1-2 cm); 95-12-2 (6-7 cm); 95-12-3 (4-5 $\mathrm{cm})$; 95-12-4 (1-2 cm):

Poor assemblages with Ericsonia subpertusa, Markalius astroporus, Cruciplacolithus tenuis, Chiasmolithus danicus, and Coccolithus cavus.

Age: Chiasmolithus danicus Zone; Early Paleocene.

Samples: 95-13-1 (26-27 cm); 95-13-2 (6-7 cm); 95-13-3 $(5.6 \mathrm{~cm})$ :

These samples contain only coccolith fragments, no identifiable species.

Sample 95-13-4 (6-7 cm):

Moderately good assemblage of corroded coccoliths, with Watznaueria barnesae, Prediscosphaera cretacea, Cribrosphaera sp.

Age: Late Cretaceous.

Samples: $95-14-1(42-43 \mathrm{~cm}) ; 95-15-1(6-7 \mathrm{~cm})$ :

Moderately good assemblage with Watznaueria barnesae, Prediscosphaera cretacea, Cribrosphaera ehrenbergi, Eiffellithus turriseiffeli, Microrhabdulus decoratus, Ahmuellerella octoradiata, and Lithastrinus grilli.

Age: Probably Campanian.
Samples: 95-15-2 (8-9 cm); 95-15-3 (7-8 cm); 95-15-4 (7-8 $\mathrm{cm})$; $95-15-5(7-8 \mathrm{~cm})$; 95-15-6 (6-7 cm); 95-16-1 (6-7 cm); 95-16-2 (6-7 cm); 95-16-3 (7-8 cm); 95-16-4 (6-7 cm); 95-16-5 (4-5 cm); 95-16-6 (6-7 cm):

Moderately good to mediocre to poor assemblages with the same species as in Sample 95-15-1 (6-7 cm).

Age: Probably Campanian.

Sample 95-17-1 $(6-7 \mathrm{~cm})$ :

Very poor assemblage with only coccolith fragments.

Samples: 95-17-2 (6-7 cm); 95-17-3 (6-7 cm); 95-17-4 (5-6 $\mathrm{cm})$; 95-17-5 (6-7 cm); 95-17-6 $(5-6 \mathrm{~cm})$ :

Moderately good assemblages like Sample 95-16-6 (6-7 $\mathrm{cm})$, but with abundant Lithraphidites carniolensis. Age: Probably Santonian.

\section{HOLE 96}

Sample 96-1-2 $(6-7 \mathrm{~cm})$ :

Very poor sample with a few reworked specimens of Watznaueria barnesae.

Sample 96-1-3 $(6-7 \mathrm{~cm})$ :

No calcareous nannofossils.

Samples: 96-1-4 $(6-7 \mathrm{~cm})$; 96-1-5 $(6-7 \mathrm{~cm})$ :

Very poor samples with a few reworked Cretaceous species, including Watznaueria barnesae, Prediscosphaera creatcea, and Kamptnerius sp.

Sample 96-1-6 (13-14 cm):

Poor assemblage of reworked Cretaceous coccoliths: Micula staurophora, Watznaueria barnesae, Prediscosphaera creatcea, and Cribrosphaera sp.

Samples: 96-2-1 (6-7 cm); 96-2-2 (6-7 cm):

Good assemblages with Cyclococcolithina foridana, Coccolithus eopelagicus, Sphenolithus moriformis, $S$. heteromorphus, Helicopontosphaera paralleta, Reticulofenestra bisecta, Discoaster nephados, D. deflandrei, and Triquetrorhabdulus carinatus.

Age: Mixed Early Miocene.

Samples: 96-2-3 (6-7 cm); 96-2-4 (6-7 cm); 96-2-5 (6-7 $\mathrm{cm})$; 96-2-6 (6-7 cm):

Good assemblages with Sphenolithus ciperoensis, $C y$ clococcolithina floridana, Coccolithus eopelagicus, Helicopontosphaera truncata, Triquetrorhabdulus carinatus, and Discoaster saundersi.

Age: Sphenolithus ciperoensis Zone; Late Oligocene.

Samples: 96-3-1 (6-7 cm); 96-3-2 (6-7 cm); 96-3-3 (6-7 cm); 96-3-4 (6-7 cm):

Moderately good assemblages with Ericsonia subpertusa, Discoaster multiradiatus, Sphenolithus anarrhopus, Coccolithus eopelagicus.

Age: Discoaster multiradiatus Zone; Late Paleocene.

Samples: 96-3-5 (6-7 cm); 96-3-9 $(6-7 \mathrm{~cm})$ : $\mathrm{cm})$

Mediocre samples with species as in Sample 96-3-1 (6-7

Age: Discoaster multiradiatus Zone; Late Paleocene.

Sample 96-5-1 $(44-45 \mathrm{~cm})$ :

Poor assemblage with Ericsonia subpertusa, Coccolithus cf. pelagicus, and Fasciculithus tympaniformis. Age: Probably Fasciculithus tympaniformis Zone; Middle Paleocene. 


\section{HOLE 97}

Samples: 97-1-1 (91-92 cm); 97-1-2 (6-7 cm); 97-1-2 (59-60 $\mathrm{cm})$ :

Good assemblages with Gephyrocapsa oceanica, $G$. caribbeanica, G. aperta, Helicopontosphaera kamptneri, and Cyclococcolithina leptopora.

Age: Gephyrocapsa oceanica Zone; Late Pleistocene.

Samples: 97-1-3 $(7-8 \mathrm{~cm})$; 97-1-4 $(6-7 \mathrm{~cm})$ :

Good assemblages with species as above, but with Pseudoemiliania lacunosa.

Age: Gephyrocapsa oceanica Zone; Late Pleistocene.

Samples: 97-2-1 (26-27 cm); 96-2-2 (6-7 cm); 96-2-3 (6-7 $\mathrm{cm})$; $96-2-4(13-14 \mathrm{~cm})$ :

Moderately good assemblages with Gephyrocapsa caribbeanica, Sphenolithus abies, Discoaster brouweri, $D$. pentaradiatus, and D. surculus.

Age: Discoaster surculus Zone; Late Pliocene.

Sample 97-3-2 $(6-7 \mathrm{~cm})$ :

Rich asterolith assemblage, with Discoaster brouweri, $D$. variabilis, $D$. cf. challengeri, and $D$. neohamatus.

Age: Discoaster calcaris Zone; Late Miocene.

Sample 97-3-3 (12-13 cm):

Poor assemblage, but with species as immediately above.

Sample 97-4-2 $(6-7 \mathrm{~cm})$ :

Mixed assemblage with Discoaster brouweri, D. variabilis, Sphenolithus heteromorphus, and Cyclococcolithina floridana.

Age: Mixed Early and Middle or Late Miocene.

Samples: 97-4-2 (58-59 cm); 97-4-3 (5-6 cm); 97-4-4 (5-6 $\mathrm{cm})$; 97-4-5 $(6-7 \mathrm{~cm})$ :

Good assemblage with Cyclococcolithina floridana, Reticulo fenestra bisecta, Sphenolithus predistentus, and $S$. distentus.

Age: Spenolithus predistentus Zone; Middle Oligocene.
Sample 97-5-1 (123-124 cm):

Good assemblage with Reticulofenestra umbilica, Coccolithus eopelagicus, Peritrachelina joidesa, and Bramlettius serraculoides.

Age: Late Eocene.

Sample 97-6-1 $(49-50 \mathrm{~cm})$ :

Very poor assemblage with Watznaueria barnesae.

Sample 97-7-1 (108-109 cm): No recognizable coccoliths.

Sample 97-7-2 (10-11 cm): Very poor assemblage with Watznaueria barnesae.

Sample 97-8-1 (74-75 cm):

Poor assemblage with Watznaueria barnesae the only recognizable species.

Sample 97-8-2 (23-24 cm):

Moderately good assemblage with Watznaueria barnesae, Eiffellithus turriseiffeli, Prediscosphaera cretacea, Ahmuellerella octoradiata, Glaukolithus diplogrammus and Staurolithites matalosus.

Age: Probably Cenomanian.

Sample 97-8-3 $(31-32 \mathrm{~cm})$ :

Poor assemblage, but with species as immediately above.

Sample 97-8-4 $(11-12 \mathrm{~cm})$ :

No recognizable calcareous nannofossils.

Sample 97-12-1 (30-31 cm):

Good assemblage like that in Sample 97-8-2 $(23-24 \mathrm{~cm})$

Age: Probably Cenomanian.

\section{REFERENCES}

Cepek, P. and Hay, W.W., 1969. Calcareous nannoplankton and biostratigraphic subdivision of the Upper Cretaceous. Trans. Gulf Coast Assoc. Geol. Soc. 19, 323.

Martini, E., 1970. Standard Paleogene calcareous nannoplankton zonation. Nature. 225, 289. 